http://www.doi.org/10.14718/ACP.2021.24.2.1

Editorial in memoriam

\title{
Alfredo Ardila. Más allá de Broca y Wernicke
}

\author{
Laura Andrea León Anhuaman*
}

Alfredo Ardila, uno de los más reconocidos neuropsicólogos de Colombia, dedicó más de 40 años de su vida a la investigación de los procesos neuropsicológicos, principalmente aquellos asociados a los procesos lingüísticos, el aprendizaje, la memoria y la producción del lenguaje. Además, muchas de sus investigaciones se centraron en la caracterización del desarrollo ontogenético del lenguaje, incluidas sus variables protectoras, facilitadoras o posibles vulnerabilidades para el desarrollo satisfactorio del lenguaje, así como la evaluación de los mecanismos que podrían estar asociados con el deterioro de las funciones lingüísticas y las patologías asociadas.

Como es sabido, las áreas y los principales procesos del lenguaje se enseñan en los primeros semestres de psicología y de carreras como fonoaudiología, y todos los que hemos pasado por esas clases hemos escuchado desde muy temprano qué son fundamentalmente el área de Broca en el lóbulo frontal y el área de Wernicke en el temporal. Por ejemplo, sabemos que Broca se ocupa principalmente de la planeación de los movimientos necesarios para la producción del lenguaje, que Wernike se encarga de la comprensión del lenguaje, y que ambas fueron delimitadas y descritas por los médicos que les dieron el nombre; asimismo, sabemos que sus primeros estudios se desarrollaron a mediados del siglo XVIII, y que hoy en día sigue existiendo un interés por delimitar estas y otras estructuras cerebrales involucradas con este proceso tan desarrollado en los humanos.

En el artículo de Ardila et al. (2016), a propósito del legado del Dr. Ardila, se hace un recuento de las áreas que han sido delimitadas como estructuras esenciales, así como de los modelos de organización del lenguaje que componen. Asimismo, se describen los patrones observados en la organización cerebral de diferentes aspectos de la producción y comprensión del lenguaje a través de distintas técnicas neurocientíficas, como la tractografía o la resonancia magnética, que permiten una delimitación más nítida de las diferentes estructuras cerebrales involucradas en el lenguaje, en comparación con las caracterizaciones previas que se hacían basadas en las lesiones de pacientes con patologías asociadas al lenguaje.

No obstante, Ardila y sus colaboradores postulan que Broca y Wernicke, a pesar de ser áreas fundamentales, son solamente parte de una red de conexiones que procesan el lenguaje y que contiene otras áreas no tan ampliamente conocidas. A partir de una metodología conocida como modelamiento de conectividad meta-analítica, los autores revisaron varios metaanálisis de investigaciones en las que los participantes desarrollaban tareas lingüísticas mientras se tomaban imágenes de resonancia magnética funcional. De acuerdo con la metodología utilizada, se puede deducir que hay conexiones entre diferentes áreas involucradas en diversas tareas de lenguaje, pues estas se activan simultáneamente mientras se está desarrollando una tarea lingüística. Incluso, en el artículo se revisan las áreas de Broadman ( $\mathrm{AB}$ ) que se activan al realizar tareas lingüísticas y que complementan las funciones de las áreas previamente descritas.

Finalmente, en la discusión del artículo se propone un modelo general de la organización de las áreas dedicadas al lenguaje, en el cual se identifican tres redes: la primera, asociada a la comprensión del lenguaje, que incluye el área de Wernicke ( $\mathrm{AB} 21$ у АB22), pero también el área auditiva primaria $(\mathrm{AB} 41$ y $\mathrm{AB} 42)$ y la circunvolución supramarginal ( $\mathrm{AB} 40)$; la segunda, en la que se incluye el área de Broca (AB44 y AB45) y las áreas BA46, BA47 y BA13; y la tercera, una parte de la corteza de la ínsula, que puede jugar un papel muy importante en la comunicación de las otras redes.

El artículo en general es un trabajo riguroso de metaanálisis que permite generar un modelo más preciso de las áreas vinculadas al lenguaje y que no solo tiene en cuenta los daños de la estructura para delimitar la función de ciertas áreas cerebrales en un proceso complejo como es el lenguaje, sino que además estudia la activación cerebral en tareas lingüísticas realizadas por personas sanas como una forma de entender el funcionamiento y organización de las diferentes áreas cerebrales involucradas en este proceso.

Universidad Sergio Arboleda. laura.leon@usa.edu.co. https://orcid.org/0000-0002-5573-7750 
Los investigadores como Alfredo Ardila dejan en sus lectores y estudiantes la disciplina y la rigurosidad como herramientas para construir y dejar conocimiento al servicio de la sociedad. De esta forma, la descripción de estos modelos permite diagnósticos muchos más precisos y formas de terapia dirigidas a daños específicos.

\section{Referencias}

Ardila, A., Bernal, B., \& Rosselli, M. (2016). How localized are language brain areas? A review of Brodmann areas involvement in oral language. Archives of Clinical Neuropsychology, 31(1), 112-122. https://doi.org/10.1093/ arclin/acv081 